\title{
Skewness as measure of the invariance of instantaneous renormalized drop diameter distributions - Part 2: Orographic precipitation
}

\author{
M. Ignaccolo and C. De Michele \\ DIIAR, Politecnico di Milano, Milano, Italy \\ Correspondence to: M. Ignaccolo (m.ignaccolo@ diiar.polimi.it)
}

Received: 2 August 2011 - Published in Hydrol. Earth Syst. Sci. Discuss.: 29 August 2011

Revised: 16 December 2011 - Accepted: 19 December 2011 - Published: 3 February 2012

\begin{abstract}
Here we use the skewness parameter, and the procedure developed in the companion paper (Ignaccolo and De Michele, 2012), to investigate the variability of instantaneous renormalized spectra of rain drop diameter in presence of orographic precipitation. Disdrometer data, available at Bodega Bay and Cazadero, California, are analyzed either as a whole, or as divided (using the bright band echo) in precipitation intervals weakly and strongly influenced by orography, and compared to results obtained at Darwin, Australia. We find that also at Bodega Bay and Cazadero exists a most common distribution of the skewness values of instantaneous spectra of drop diameter, but peaked at values greater than 0.64, found at Darwin. No appreciable differences are found in the skewness distributions of precipitation weakly and strongly influenced by orography. However the renormalized drop diameter spectra of precipitation with strong orographic component have fatter right tail than precipitation with a weaker orographic component. The differences between orographic and non-orographic precipitation are investigated within the parametric space represented by number of drops, mean value and standard deviation of drop diameter. A filter is developed which is able to identify $1 \mathrm{~min}$ time intervals during which precipitation is mostly of orographic origin.
\end{abstract}

\section{Introduction}

Stratiform and convective are the two main categories used to describe rainfall. They indicate the strength (convective indicating the stronger case) of the updraft motion generating atmospheric vapor condensation and eventually rain drops
(Houze, 1997). This categorization does not include explicitly the orography: the interaction between the land surface and the atmosphere. Mountains, and to a lesser degree hills, generally induce an uplift motion which may produce condensation and then precipitation (Roe, 2005). One of the most important effects due to orography is the so called "orographic enhancement": the total amount of rainfall on the slopes of mountains and hills is larger than that on the surrounding planes. However, the focus of this manuscript will be the effect of orography on the shape of drop diameter distributions regardless of any effect on cumulated rainfall rates.

In the present manuscript we consider data sets from three different sites: (1) Darwin (DRW), Australia, (2) Bodega Bay (BBY), California, USA, (3) Cazadero (CZD), California, USA. Darwin is on the coast backed by plains and it can be safely assumed the land surface "influence" on rain (intended as disturbance on rainfall rates, drop size distributions, and all the possible features of the rainfall phenomenon) is negligible. This data set is the same one used in the companion paper "Skewness as measure of the invariance of instantaneous renormalized drop diameter distributions - Part 1: Convective vs. stratiform precipitation" (Ignaccolo and De Michele, 2012). Bodega Bay is located on the coast of California, $\sim 120 \mathrm{~km}$ North of San Francisco, while Cazadero is $10 \mathrm{~km}$ inland, $33 \mathrm{~km}$ Northwest of Bodega Bay. Immediately west of Bodega Bay are located the Coastal Range Mountains of California where Cazadero is at $475 \mathrm{~m}$ a.s.m.l. (all peaks in the region surrounding Bodega Bay and Cazadero are below $1000 \mathrm{~m}$ of altitude). At BBY and CZD the land surface influence on precipitation can be relevant. As matter of fact it has been observed that winter stratiform storms at these sites may lack the characteristic bright band echo signature

Published by Copernicus Publications on behalf of the European Geosciences Union. 
for extensive period of times (White at al., 2003; Martner et al., 2008). During these time intervals, precipitation "is primarily the result of orographically forced condensation and coalescence processes" (Martner et al., 2008).

It is important to stress that the distinction between time intervals where the bright band echo is present (BB intervals) versus those in which it is absent (NBB intervals) is done averaging $30 \mathrm{~min}$ radar data, and it is not intended as a distinction between not orographic versus orographic rain ("BB and NBB rain often occurs in superposition", Martner et al., 2008) but more as the predominance of one mechanism of drop formation over another: BB mostly stratiform and NBB mostly orographic. The coastal range mountains in BBY and CZD can always induce condensation and coalescence of water droplets. Therefore the mere presence of the bright band signature does not imply automatically the absence of precipitation of orographic origin. Conversely, the absence of the bright band signature does not automatically imply that aggregation of snow/ice crystals (the typical mechanism of drop production of stratiform rain) is absent but that (1) it is not so widespread for the melting of snow/ice crystals to produce a strong bright band echo, (2) it is not predominant with respect to the precipitation of orographic origin.

The main purpose of this manuscript is to investigate the variability of the instantaneous (1 min sampling interval) renormalized spectra, in orographic precipitation, making use of the skewness. In the companion paper (Ignaccolo and De Michele, 2012) we have investigated the variability at Darwin for the entire dataset and the stratiform and convective subsets. Hereby this investigation is repeated for the Bodega Bay and the Cazadero datasets in their entirety and dividing each of these in two subsets: BB (bright band echo present) and NBB (bright band echo absent). Moreover a comparison is made between the results at Darwin and those at Bodega Bay and Cazadero.

As in the companion paper (Ignaccolo and De Michele, 2012), we divide each dataset in subsets of comparable values of skewness: skewness classes. For each skewness class subset, we compare the renormalized spectra for BB and NBB rain periods. While in Darwin stratiform (BB) and convective (NBB) precipitation have the same renormalized spectra, this is not true for Bodega Bay and Cazadero as NBB 1 min time intervals have fatter right tails with respect to $B B$ 1 min time intervals. Prior to renormalization instantaneous NBB spectra are much steeper (as identified by the value of the gradient of the distribution around its maximum) than BB spectra. We use this property to build a steepness-orographic filter which labels 1 min time intervals as steep or not-steep. Steep time intervals are time intervals during which precipitation is mostly orographic in origin, and "generate" the fatter right tail observed in renormalized spectra of NBB data sets (i.e. removing steep time intervals from the NBB data sets removes the discrepancies between the renormalized spectra of BB and NBB skewness class subsets).

\section{Data and methods}

Here we summarize the data processing and methods adopted in our analysis. Most of these are the same as those used in the companion paper (Ignaccolo and De Michele, 2012), in which case we present only a "quick" summary (the interested reader will find in the companion paper all the details), while new methods will be described in detail.

\subsection{Data}

We use Joss Waldvogel RD80 disdrometer data at $1 \mathrm{~min}$ time resolution from three different locations (1) Darwin (DRW), Australia (12.45 $\mathrm{S}, 130.83^{\circ} \mathrm{E}, 2 \mathrm{~m}$ a.m.s.l.), (2) Bodega Bay (BBY), CA, USA $\left(38.32^{\circ} \mathrm{N}, 123.07^{\circ} \mathrm{W}\right.$, $12 \mathrm{~m}$ a.m.s.l.), (3) Cazadero (CZD), CA, USA $\left(38.61^{\circ} \mathrm{N}\right.$, $123.21^{\circ} \mathrm{W}, 475 \mathrm{~m}$ a.m.s.l.). For all datasets the drop diameters are classified in 20 different classes covering the range $0.3-5.6 \mathrm{~mm}$, and 1-min counts are corrected against the instrument dead time (Sauvageot and Lacaux, 1995). The Darwin data set is the same as in the companion paper (Ignaccolo and De Michele, 2012). It consists of 97 consecutive days of measurements, from 4 November 2005 to 10 February 2006. Reflectivity maps are available for the time intervals 9 November to 6 December 2005, and 6 January to 10 February 2006, allowing for stratiform versus convective classification through the identification of the bright band. A total of 19 stratiform and 33 convective time intervals were identified with this method (see the online material in Ignaccolo and De Michele, 2010). The BBY data set consists of 1 min disdrometer counts for 100 non consecutive days spanning the 2003/2004 winter season from 6 December to 25 March. The CZD data set consists of 1 min disdrometer counts for 375 non consecutive days covering the winter seasons from year 2003 to 2006 . Winter maritime storms at BBY and CZD are stratiform in nature. However radar data collected during these storms have shown that the "bright band" echo, which is characteristic of stratiform precipitation, is missing during extended period of times, not only at BBY and CZD, but on other locations in the Pacific North American coast as well (Neiman et al., 2005). The lack of the bright band has been associated (White at al., 2003; Martner et al., 2008) to time intervals of hydrometeor growth primarily resulting of condensation and coalescence of water droplets in a relatively shallow layer near the surface: a mechanism of orographic origin (Kingsmill et al., 2006). From the BBY data set and the 2003/2004 winter part of the CZD database, we consider time intervals where the bright band is present (BB) and time intervals where the bright band is absent (NBB) following the classification scheme of Table I of Martner et al. (2008). In this way the subsets BBYBB, BBY-NBB, CZD-BB, and CZD-NBB are obtained.

These data are processed as in the companion paper (Ignaccolo and De Michele, 2012). Firstly minutes with total counts $<=60$, or with only 2 diameter classes with non zero 
counts, are eliminated from the database. This procedure ensures that mean, standard deviation and other variables used to describe instantaneous distributions are statistically significant. After this procedure the remaining 1 min intervals are checked for outliers drop counts. Outliers drop counts occur when gaps (disdrometer classes with zero counts) exist between occupied diameter classes. For each minute we identify the class with the maximum count, and then the no gap region containing it. Drop counts which do not belong to the no gap region constitute an extremely small portion of the database $(\sim 0.1-0.2 \%$ of the total number of drops) but they can produce statistical artifacts (see Appendix), and thus these drop counts are set to zero. Once processed as described above, the three data sets considered in this work and their subsets have the following compositions: (1) DRW $6863 \mathrm{~min}$ and 2753796 drops, DRW stratiform $1844 \mathrm{~min}$ and 354743 drops, and DRW convective $1536 \mathrm{~min}$ and 1064561 drops. (2) BBY $10804 \mathrm{~min}$ and 5389240 drops, BBY-BB 3273 and 1493843 min, BBYNBB 2614 minu and 1517381 drops. (3) CZD $76137 \mathrm{~min}$ 44252384 drops, CZC-BB $3883 \mathrm{~min}$ and 2006234 drops, and CZC-NBB $3551 \mathrm{~min}$ and 2887789 drops.

\subsection{Methods}

We adopt the same renormalization procedure of the companion paper (Ignaccolo and De Michele, 2012). For each renormalization time interval $I, 1 \mathrm{~min}$ in our case:

$$
\left\{\begin{array}{l}
D \rightarrow D_{R}=\frac{D-\mu_{I}}{\sigma_{I}} \\
p_{G, I}(D) \rightarrow p_{G, I}\left(D_{R}\right)=\sigma_{I} p_{G, I}\left(\sigma_{I} D_{R}+\mu_{I}\right)
\end{array}\right.
$$

where $\mu_{I}$ and $\sigma_{I}$ are the mean, and standard deviation of the drop diameter observed at the ground. This renormalization, originally introduced in (Ignaccolo et al., 2009; Ignaccolo and De Michele, 2010), operates differently from the other renormalizations available in Literature, e.g. (Willis, 1984; Sempere Torres et al., 1994; Maki et al., 2001; Testud et al., 2001; Uijlenhoet et al., 2003; Campos et al., 2006; Hazenberg et al., 2011). In fact Eq. (1) operates a change of variable, from the drop diameter $D$ to the renormalized diameter $D_{R}$, having zero mean and unit variance. The instantaneous renormalized spectrum is, in this case, the instantaneous probability density $p_{G, I}\left(D_{R}\right)$ of the renormalized drop diameter. This density is obtained from that of the drop diameter observed at the ground $p_{G, I}(D)$ using the second identity of Eq. (1).

Usually disdrometer data are categorized in diameter classes so that for each drop one does not know the "exact" value of the diameter, but only that the diameter was in a given range. We refer to this effect as the "quantization error". Due to the quantization error the probability density $p_{G, I}(D)$, and as a consequence the probability density $p_{G, I}\left(D_{R}\right)$, is a step function with constant value inside the range of each diameter class. Moreover given a series of disdrometer counts, it is not possible to associate to it a unique series of renormalized diameter $D_{R}$. However one can associate to a disdrometer count $n_{j}$ of the $j$-th class, $n_{j}$ different diameter values $D$ extracted randomly and uniformly in the range of $j$-th class, and using the first identity of Eq. (1) $n_{j}$ different values of the renormalized drop diameter $D_{R}$. With this process of "randomization", one can associate to a sequence of disdrometer drop counts a sequence of renormalized drop diameters, and then a density $p_{G}^{\text {rand }}\left(D_{R}\right)$. Each repetition of the randomization process creates a new sequence and a new density. In the limit of an infinite number of repetitions the arithmetic average of the densities $p_{G}^{\text {rand }}\left(D_{R}\right)$ converges to a limiting distribution $p_{G}\left(D_{R}\right)$ :

$p_{G}\left(D_{R}\right)=\frac{1}{N} \sum_{I} N_{I} p_{G, I}\left(D_{R}\right)$

where $N_{I}$ is the drop count in the $I$-th renormalization time interval, $p_{G, I}\left(D_{R}\right)$ is the instantaneous renormalized spectrum, and $N$ is the total number of drops in the particular sequence of disdrometer drop counts considered. We consider the probability density $p_{G}\left(D_{R}\right)$ defined by Eq. (2) as the renormalized spectrum associated with a particular series of disdrometer drop counts.

As in the companion paper (Ignaccolo and De Michele, 2012), the skewness is used to compare instantaneous renormalized spectra $p_{G, I}\left(D_{R}\right)$ and the concept of skewness class will be used to divide a data set in subsets with comparable values of skewness. A renormalization time interval $I$ belongs to the skewness class $r$ if the relative difference of the corresponding skewness $\gamma_{I}$ with respect value 0.64 is within the percentage range $[(r-1 / 2) \times 100$, $(r+1 / 2) \times 100$ ]: e.g. the skewness class zero (s0) implies $\gamma_{I}$ in the range $[0.32,0.96]$, the skewness class plus-one $(s+1)$ implies $\gamma_{I}$ in the range [0.96, 1.60], while the skewness class minus-two $(\mathrm{s}-2)$ implies $\gamma_{I}$ in the range $[-0.96,-0.32]$. The value 0.64 is adopted as reference for the skewness parameter in order to have the same skewness classes as the companion paper (Ignaccolo and De Michele, 2012) and in order to compare results at different sites (DRW, BBY, and CZD) for a fixed skewness class.

\section{Additional parameters used to describe drop size distributions}

Additional parameters are used to characterize the properties of the instantaneous spectra. The first additional parameter is the kurtosis $k_{I}$ (the fourth standardized moment) since it is the next logical choice after the skewness $\gamma_{I}$, in the statistical description of a distribution through its central standardized moments. We remind the reader that instantaneous not renormalized and renormalized spectra have the same value of all standardized moments of order $\geq 3$, (skewness, kurtosis, etc.) in virtue of Eq. (9) of the companion paper (Ignaccolo and De Michele, 2012). In addition to the kurtosis we will use four additional parameters. These parameters aim to "capture" 
what are the known properties of orographic not renormalized instantaneous drop size distributions $\left(p_{G}^{I}(D)\right)$ : (1) abundance of small drops with the largest diameter rarely exceeding $2 \mathrm{~mm}$ (narrow) (Blanchard, 1953; Fujiwara, 1967), (2) widely skewed (Campos, 1999) with a steep exponential decay at larger diameters (Martner et al., 2008). The four parameters are: (1) the disdrometer diameter class of maximum density $j_{m, I}$ : the modal class. (2) The class span $S_{I}$ : the number of consecutive disdrometer diameter classes with non zero probability density. (3) The percentage occupancy $\% O_{I}$ : the fraction $(0 \rightarrow 1)$ of the probability density contained in the first four disdrometer diameter classes. (4) The left and right gradients $\nabla_{L, I}$ and $\nabla_{R, I}$ of the probability density around the maximum value. These last quantities are defined as follows:

$$
\left\{\begin{array}{l}
\nabla_{L, I}=\frac{p_{G, I}\left(D_{j_{m, I}-k}\right)-p_{G, I}\left(D_{j_{m, I}}\right)}{D_{j_{m, I}}^{R}-D_{j_{m, I}-k}^{L}} \\
\nabla_{R, I}=\frac{p_{G, I}\left(D_{j_{m, I}+k}\right)-p_{G, I}\left(D_{j_{m, I}}\right)}{D_{j_{m, I}+k}^{R}-D_{j_{m, I}}^{L}}
\end{array}\right.
$$

where $D_{j_{m, I}}$ is the middle value of the $j_{m, I}$ diameter class, while the symbol $D_{l}^{L}\left(D_{l}^{R}\right)$ indicates the left (right) border of the $l$ diameter class. The integer $k$ is set to 4 unless $p_{G, I}\left(D_{j_{m, I} \pm 4}\right)$ is equal to zero or $j_{m, I} \pm 4$ is not a valid value $([1,20])$ for a diameter class. In such a case $k$ is set to the maximum possible value in the range $[0,3]$ so that $p_{G, I}\left(D_{j_{m, I}} \pm 4\right)$ is larger than zero or $j_{m, I} \pm 4$ is a valid value for a class diameter: e.g. if $j_{m, I}=0$ than $k=0$ and $\nabla_{L, I}=0$.

\section{Results}

\subsection{Skewness}

Figure 1 shows the probability $\operatorname{Pr}\left(\gamma_{I}\right)$ of having a renormalized (not renormalized) spectrum $p_{G, I}\left(D_{R}\right)\left(p_{G, I}(D)\right)$ with skewness $\gamma_{I}$ for all the datasets examined: DRW database and its two subsets, stratiform and convective, on the top panel, BBY and its two subsets, BBY-BB and BBY-NBB, on the middle panel, and CZD and its two subsets, CZD-BB and CZD-NBB, on the bottom panel. The results in Bodega Bay and Cazadero mirror those in Darwin. In all cases the probability $\operatorname{Pr}\left(\gamma_{I}\right)$ for the entire datasets (solid lines) is strongly peaked. In all cases the distributions relative to subsets with a bright band signature (DRW stratiform, BBY-BB, and CZD$\mathrm{BB}$ ) and to those without a bright band signature (DRW convective, BBY-NBB, and CZD-NBB) are similar to each other and to the results for the entire datasets. The main difference is in the locations of the peaks, the distributions in Darwin are peaked at $\gamma_{I} \simeq 0.64$, those in Bodega Bay at $\gamma_{I} \simeq 0.72-0.88$, and those in Cazadero at $\gamma_{I} \simeq 0.88-1.04$. We will propose an explanation for the possible origin of the shift in the peak location in the "Conclusions" Section.

Table 1 reports, for bright band and non bright band subsets at Bodega Bay and Cazadero, the percentages I\% of
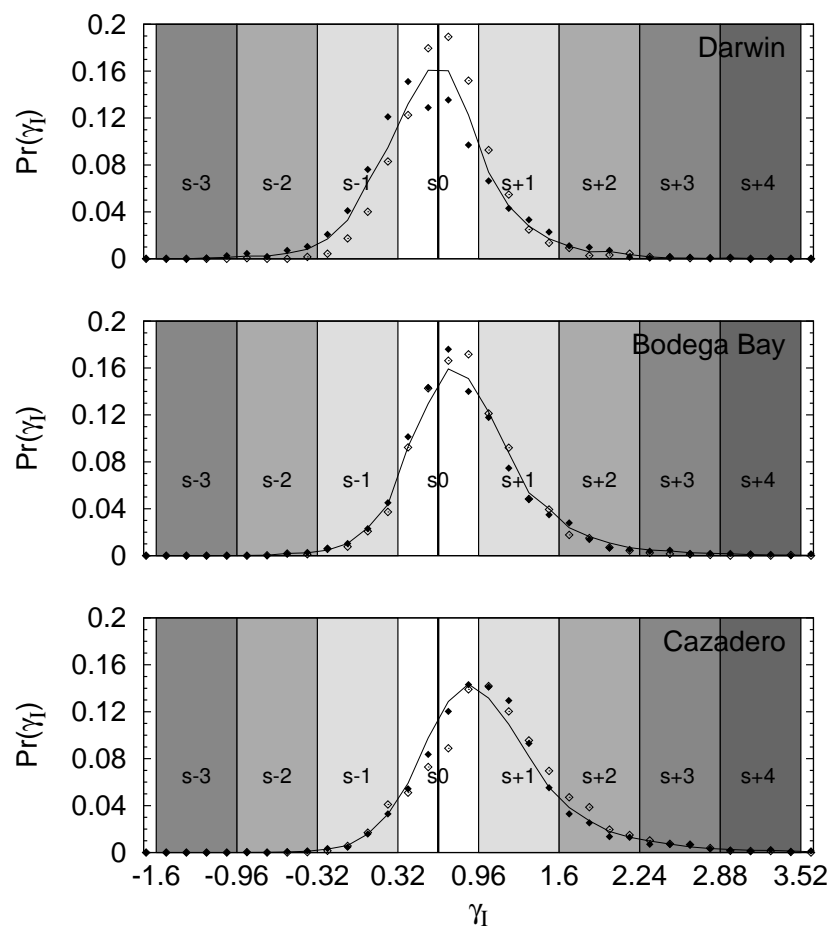

Fig. 1. The probability $\operatorname{Pr}\left(\gamma_{I}\right)$ for the datasets examined. In all panels, solid lines indicate the probability for the entire dataset, white diamonds the probability for subsets with bright band signature (DRW stratiform, BBY-BB, and CZD-BB), and black diamonds the probability for subsets without a bright band signature (DRW convective, BBY-NBB, and CZD-NBB). The shaded regions indicate different skewness classes.

the renormalization time intervals in the data set belonging to a given skewness class, and the percentage $d \%$ of the database total number of drops belonging to renormalization time intervals in a given skewness class. As expected from Fig. 1, the percentage $I \%$ inside each skewness class does not change appreciably between BB and NBB subsets in the same location, the only notable differences are $\sim+5 \%$ difference in the population of the s0 skewnwss class, and $\sim-4 \%$ in the population of the $\mathrm{s}+2$ skewness class between CZD$\mathrm{BB}$ and CZD-NBB. More notable differences occur if one considers the $d \%$ of drops inside each skewness class. In this case when moving from the BB to the NBB subset the percentage of drops in the skewness class s0 increases $(\sim+7.5 \%$ for BBY and $\sim+10 \%$ for CZD) and that in the skewness class $\mathrm{s}+2$ decreases $(\sim-2.5 \%$ for BBY and $\sim-6.5 \%$ for CZD).

\subsection{Renormalized spectra of skewness class data sets}

Next, we divide, as done in the companion paper (Ignaccolo and De Michele, 2012), all databases in subsets according to the skewness class of each renormalization time interval. We calculate the probability density $p_{G}\left(D_{R}\right)$ of the renormalized 
Table 1. Classification of the BBY-BB, BBY-NBB, CZD-BB, and CZD-NBB data sets in classes of skewness using the number of time intervals $(I \%)$, and number of drops $(d \%)$.

\begin{tabular}{|c|c|c|c|c|c|c|c|c|}
\hline \multirow{2}{*}{$\begin{array}{l}\text { Data set } \\
\text { Class of } \gamma\end{array}$} & \multicolumn{2}{|c|}{ BBY BB } & \multicolumn{2}{|c|}{ BBY NBB } & \multicolumn{2}{|c|}{ CZC BB } & \multicolumn{2}{|c|}{ CZC NBB } \\
\hline & $I \%$ & $d \%$ & $I \%$ & $d \%$ & $I \%$ & $d \%$ & $I \%$ & $d \%$ \\
\hline$s-2[-0.96,-0.32]$ & 0.33 & 0.21 & 0.42 & 0.18 & 0.05 & 0.009 & 0.25 & 0.05 \\
\hline $\mathrm{s}-1[-0.32,0.32]$ & 7.15 & 7.32 & 8.49 & 5.03 & 6.51 & 5.88 & 5.66 & 3.32 \\
\hline s0 $[0.32,0.96]$ & 57.28 & 53.92 & 56.04 & 61.57 & 35.17 & 33.89 & 40.13 & 43.38 \\
\hline$s+1[0.96,1.60]$ & 30.09 & 29.82 & 27.54 & 25.84 & 42.72 & 41.21 & 41.87 & 42.51 \\
\hline$s+2[1.60,2.24]$ & 4.40 & 7.51 & 5.35 & 4.90 & 12.02 & 14.05 & 8.47 & 7.46 \\
\hline$s+3[2.24,2.88]$ & 0.58 & 0.80 & 1.22 & 1.20 & 2.83 & 3.98 & 2.29 & 2.17 \\
\hline $\mathrm{s}+4[2.88,3.52]$ & 0.12 & 0.34 & 0.46 & 0.54 & 0.46 & 0.63 & 0.59 & 0.53 \\
\hline$s+5[-0.96,-1.60]$ & 0.03 & 0.06 & 0.34 & 0.54 & 0.12 & 0.20 & 0.50 & 0.44 \\
\hline$s+6[-0.96,-1.60]$ & 0 & 0 & 0.04 & 0.07 & 0.08 & 0.12 & 0.08 & 0.09 \\
\hline
\end{tabular}

drop diameter $D_{R}$ for each subset using Eq. (2). Figure 2 depicts the results for the skewness classes from -2 to +3 (the remaining skewness classes have less than 10 time intervals in the corresponding data sets and are not considered for comparison) for the entire DRW data set (solid line), BBYBB (long dashed line) and BBY-NBB (circles) subsets, and CZD-BB (short dashed line) and CZD-NBB (triangle) subsets. For the Darwin site no distinction is made between stratiform and convective since no appreciable difference exists in this case (see Fig. 3 of the companion paper, Ignaccolo and De Michele, 2012). We see how the results for the BBY-BB and CZD-BB are similar to those of the Darwin data sets for all the skewness classes. Some differences are observed for the $\mathrm{s}+3$ skewness class where DRW, BBY-BB, and CZD$\mathrm{BB}$ densities have the same shape until the DRW and then the BBY-BB densities drop sharply. These differences are due to sampling inaccuracies and are not statistically significant. We consider the range for which densities are statistically significant as the range for which the density values correspond to probability values $\geq 10 / M, M$ being the total number of drops in the data set considered. When the densities $p_{G}\left(D_{R}\right)$ of NBB subsets are compared to BB subsets we see how a fatter left tail is present for the $\mathrm{s} 0, \mathrm{~s}+1$, and $\mathrm{s}+2$ skewness classes. These differences cannot be ascribed just to sampling effects. On the contrary, the fatter left tails for the $s+3$ class, and the lack of an extended right tail for the $\mathrm{s}-1$ and $\mathrm{s}-2$ classes are due to sampling inaccuracies.

Figure 2 indicates that the main differences between the densities $p_{G}\left(D_{R}\right)$ of the renormalized drop diameter $D_{R}$ relative to the BB and NBB subsets both at Bodega Bay and at Cazadero occur for the skewness class $\mathrm{s} 0, \mathrm{~s}+1$, and $\mathrm{s}+2$. Since during time intervals without bright band signature precipitation is mostly orographic in nature (Martner et al., 2008), the observed differences must be due to peculiar properties of the shape of instantaneous spectra of orographic origin. These properties are evidently not "captured" by the skewness parameter since Fig. 1 indicates that there are no relevant differences in the distribution of skewness values for the BB and NBB rain in both Bodega Bay and Cazadero sites. Are there some parameters other than the skewness which can be used to "capture" the BB vs. NBB discrepancies?

\subsection{Differences in spectra between BB from NBB precipitation}

We will now look for parameters describing instantaneous spectra, $p_{G, I}(D)$, to build a metric which somewhat separates bright band from not bright band rain time intervals at Bodega Bay and Cazadero. In order to avoid presenting the results of the following analysis for the $\mathrm{s} 0, \mathrm{~s}+1$ and $\mathrm{s}+2$ classes separately, we pasted together the $\mathrm{s} 0, \mathrm{~s}+1$, and $\mathrm{s}+2$ subsets creating the $\mathrm{s} 012$ skewness class subset for each one of the databases we considered: s012-BBY-BB, s012-BBYNBB， s012-CZD-BB， s012-CZD-NBB， s012-DRW stratiform, and s012-DRW convective (we did split the Darwin data set so that we can directly compare bright band data sets at Bodega Bay and Cazadero with stratiform precipitation in absence of any orographic effect as we expect to be the case in Darwin). Results relative to 5012 subsets do not differ for all practical purposes from those relative to single skewness classes ( $\mathrm{s} 0, \mathrm{~s}+1$, and $\mathrm{s}+2)$ subsets. In all the figures presented, lines will indicate the results for $\mathrm{s} 012$ subsets with a bright band signature (solid line for s012-DRW stratiform, long dashed line for s012-BBY-BB, and short dashed lines for s012-CZD-BB dataset), while points indicate the results for data sets without a bright band signature (squares for s012-DRW convective, circles for s012-BBY-NBB, triangles for the s012-CZD-NBB).

\subsubsection{Kurtosis}

The top-left panel of Fig. 3 reports the cumulative distribution functions $\mathcal{F}\left(\kappa_{I}\right)$ of the kurtosis for both the bright band and non bright band data sets. No relevant differences are observed, thus we judge the kurtosis not to be a valid metric 

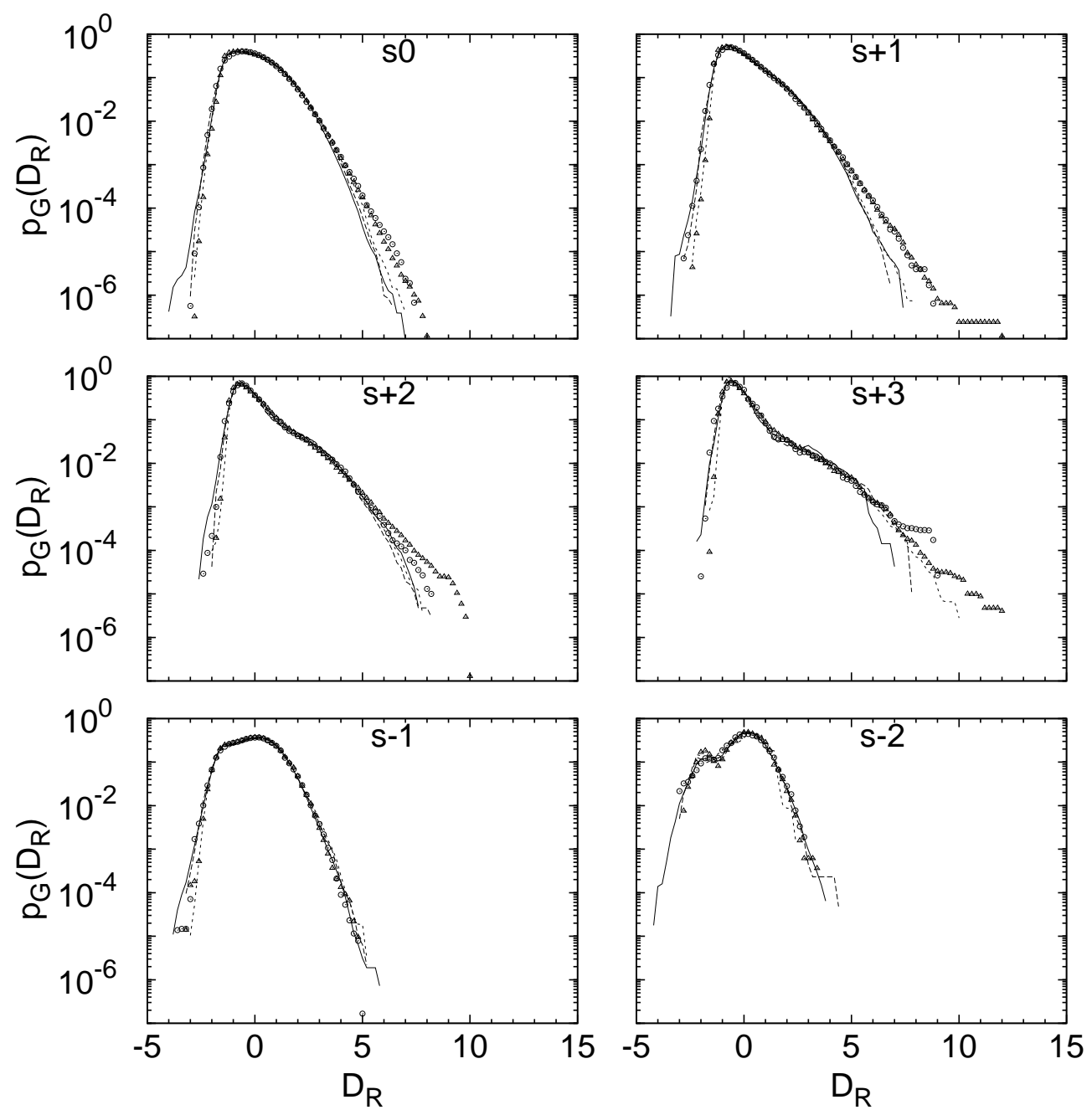

Fig. 2. $p_{G}\left(D_{R}\right)$ for skewness class subsets obtained from the entire DRW database (solid line), the BBY-BB database (long dashed line), the CZD-BB databases (short dashed line), the BBY-NBB database (circles), and the CZD-NBB database (triangles). The label on the top of each panel indicates the skewness class: $\mathrm{s} 0, \mathrm{~s}+1, \mathrm{~s}+2, \mathrm{~s}+3, \mathrm{~s}-1$, and $\mathrm{s}-2$.

to "separate" BB and NBB data sets at Bodega Bay and Cazadero. We notice how the kurtosis values grow larger moving from Darwin to Bodega Bay and then to Cazadero. This growth is simply due to the growth in skewness (shift of the peak location of $\operatorname{Pr}\left(\gamma_{I}\right)$ shown in Fig. 1) as the skewness value "fixes" also the kurtosis value. To prove this point we consider the range $[0.32,2.24]$ of skewness values (the entire range of the $\mathrm{s} 0, \mathrm{~s}+1$, and $\mathrm{s}+2$ skewness classes) and divide it in bins of width 0.16 ( $1 / 4$ of the range of a skewness class). For each bin we consider all the possible values of the kurtosis and calculate the $5 \%, 50 \%$, and $95 \%$ quantile of the corresponding distributions. We see, top-right panel and bottom panels of Fig. 3, how the 5\% and 50\% are really independent from the particular data sets considered. The same can be said for the $95 \%$ althought the results are more "noisy" as they are partially affected by statistically inaccuracies. In this sense, the skewness $\gamma_{I}$ "determines" the kurtosis $\kappa_{I}$ not depending on site location or presence/absence of a bright band radar echo.

\subsubsection{Other parameters}

Next, we consider other possible parameters describing the instantaneous spectra in order to differentiate between BB and NBB rain periods. These parameters are (see Sect. 2.2) the disdrometer diameter class of maximum density $j_{m, I}$, the class span $S_{I}$, the percentage occupancy $\% O_{I}$, the left and right gradients $\nabla_{L, I}$ and $\nabla_{R, I}$ of the probability density around the maximum value. Figure 4 illustrates the cumulative density functions for these variables. When we compare the NBB result at Bodega Bay and Cazadero with the corresponding $\mathrm{BB}$ at the same sites, we see how NBB instantaneous probability densities $p_{G, I}(D)$ are more concentrated on the first four diameter classes $\Rightarrow D \leq 0.715 \mathrm{~mm}$ $\left(\mathcal{F}\left(\% O_{I}\right)\right)$, are narrower $\left(\mathcal{F}\left(S_{I}\right)\right)$, and have the maximum 

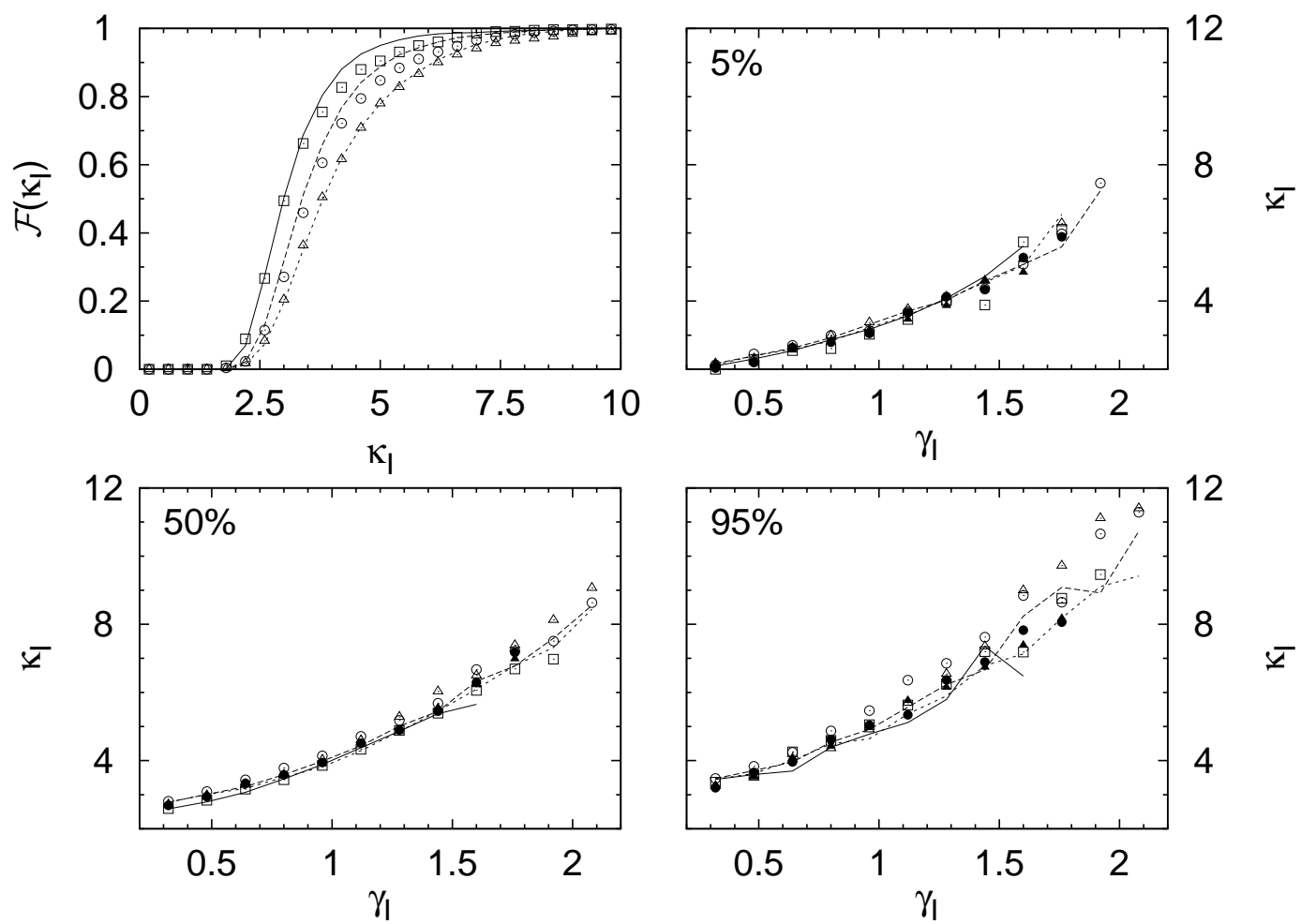

Fig. 3. Distribution of kurtosis and skewness-kurtosis relations. The top-left panel reports the cumulative distribution functions $\mathcal{F}\left(\kappa_{I}\right)$ of the kurtosis. The other three panels give the $5 \%$ (top-right panel), 50\% (down-left panel), and $95 \%$ (down-right panel) percentile of kurtosis in 0.16 width bin of skewness. The solid line represents DRW-BB database, long dashed line the BBY-BB database, short dashed line the CZD-BB database, the squares the DRW-NBB, the circles the BBY-NBB database, and triangles the CZD-NBB database.

located predominantly ( $>90 \%$ of the cases) in the first 4 diameter class $\left(\mathcal{F}\left(j_{m, I}\right)\right)$. These observed properties closely match the known properties of orographic precipitation: drop size distributions which have an abundance of small drops, and are narrow, with the largest diameters rarely exceeding $2 \mathrm{~mm}$ (Blanchard, 1953; Fujiwara, 1967). We note also how the discrepancies between NBB and BB are consistently larger in Cazadero than in Bodega Bay. When the cumulative density functions $\mathcal{F}\left(\% O_{I}\right)$ and $\mathcal{F}\left(j_{m, I}\right)$ for the s012-BBY-BB and s012-CZD-BB data sets are compared to those relative to the s012-DRW stratiform data set, we observe the same discrepancies observed in the NBB vs. BB comparison but with a "smaller" intensity. Once again for Cazadero larger differences are recorded than for Bodega Bay. These reduced discrepancies are compatible with the notion that even if the bright band signature is present precipitation which is orographic in nature may still occur (Martner et al., 2008). Regarding the span $S_{I}$ we observe a slightly larger (smaller) average value for the s012-BBY-BB (s012CZD-BB) data set when compared to the Darwin stratiform data set. Finally the results relative to the DRW convective data set show that convective precipitation with respect to stratiform precipitation has on average a larger span $S_{I}$, but comparable percentage occupancy $\% O_{I}$ and disdrometer diameter class of maximum density $j_{m, I}$.
The bottom panel in Fig. 4 shows the cumulative density functions for the left and right gradients $\nabla_{L, I}$ and $\nabla_{R, I}$ of the instantaneous probability density $p_{G, I}(D)$ around the maximum value. The horizontal solid line indicates the $5 \%$ probability level, while the vertical line indicates the zero value for both the left and right gradients. The right gradients of the NBB databases at Bodega Bay and Cazadero have much smaller $(\simeq 4$ times smaller) $5 \%$ quantile and wider range (0 to minimum value) than the corresponding BB data bases. Among data sets with a bright band s012BBY-BB and s012-CZD-BB have a much wider $(\simeq 3$ time larger) range than the DRW stratiform data set, however the $5 \%$ quantile of s012-BBY-BB is almost identical to DRW stratiform, while that of s012-CZD-BB is almost the double. In all cases, databases at Cazadero have the smallest $5 \%$ quantile. For the left gradients both s012-BBY-NBB and s012-CZD-NBB have a smaller $(-60 \%$ and $-70 \%$ resepctively) $5 \%$ quantile than s012-BBY-BB and s012-CZD-BB. The range of s012-BBY-NBB is wider than those of s012BBY-BB but the opposite is true for s012-CZD-NBB and s012-CZD-BB. Moreover the data sets in Bodega Bay are those with the smallest $5 \%$ quantile. We think, this is due to the fact that for $\gtrsim 60 \%$ of the s012-CZC-NBB data set the disdrometer diameter class of maximum density $j_{m, I}$ is the first one $\Rightarrow \nabla_{L, I}=0$, with second third and fourth classes 

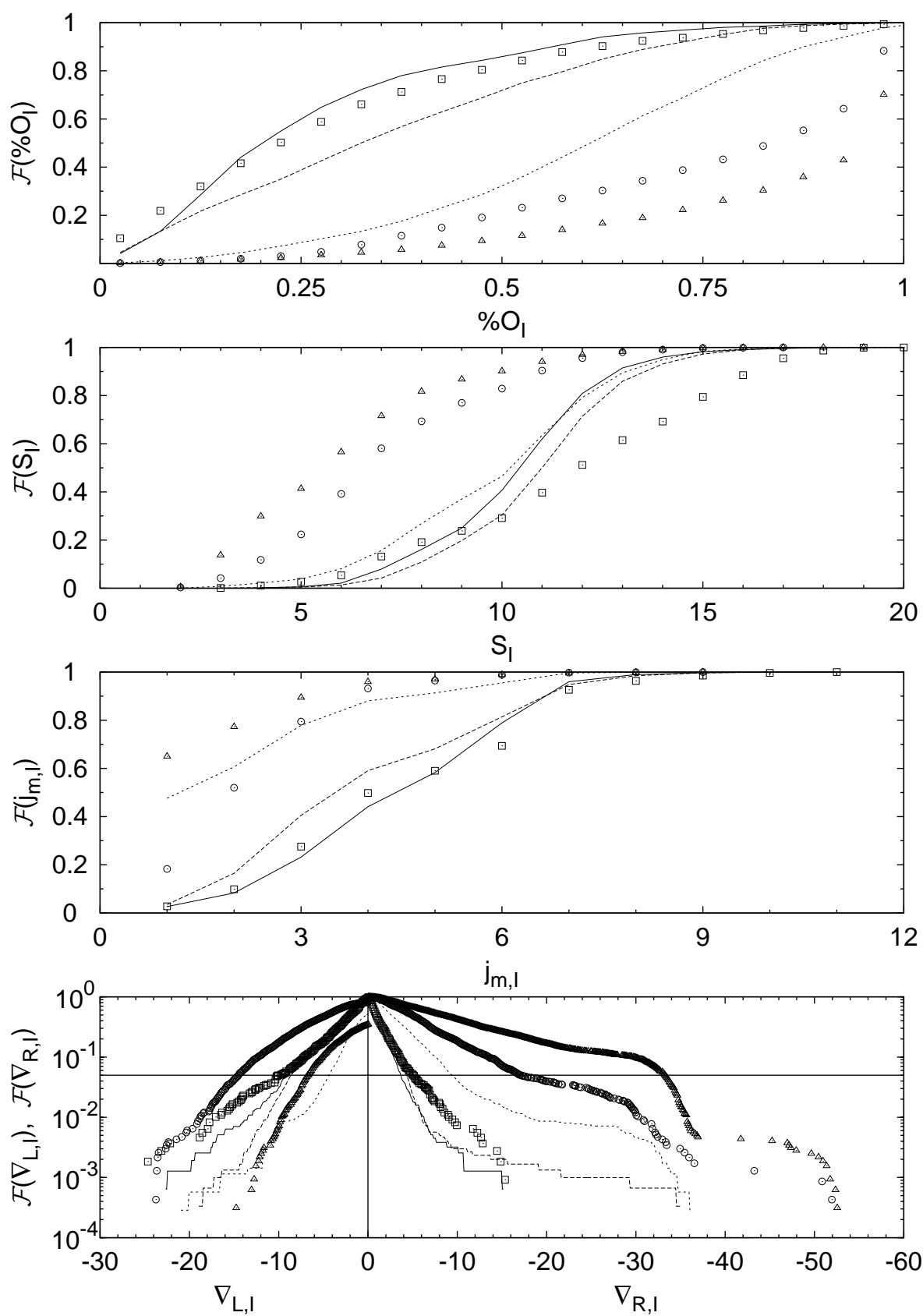

Fig. 4. Cumulative density functions of the following variables: the percentage occupancy $\% O_{I}$ (upper panel), the class span $S_{I}$ (second panel from the top), the disdrometer diameter class where the maximum density $j_{m, I}$ (third panel from the top) occurs, the left and right gradients $\nabla_{L, I}$ and $\nabla_{R, I}$ of the probability density around the maximum value (lower panel). The solid line represents DRW-BB database, long dashed line the BBY-BB database, short dashed line the CZD-BB database, the squares the DRW-NBB, the circles the BBY-NBB database, and triangles the CZD-NBB database. The horizontal solid line indicates the $5 \%$ probability level.

having a much smaller probability. However for the s012BBY-NBB the most probable disdrometer diameter class of maximum density $j_{m, I}$ is the second class followed by the third and the by the first $(\leq 20 \%)$. As a consequence there are many more $\nabla_{L, I}<0$ for s012-BBY-NBB than for s012CZD-NBB. For data sets with a bright band signature, the
s012-DRW stratiform dataset and the s012-BBY-BB have almost identical left gradients, while the s012-CZD-BB value is larger by a factor 0.5 . The range is also the widest for s012-DRW stratiform, with s012-BBY-BB and s012-CZDBB having both $\simeq 20 \%$ smaller ranges. Finally both for the right and left gradients the $5 \%$ quantile and the range of the 
s012-DRW convective data set is similar to that of the s012DRW stratiform data set.

\subsection{Steepness filter}

The division in BB versus NBB sets according to radar maps is not a "black vs. white" one. Radar maps are obtained averaging $30 \mathrm{~min}$ radar reflectivity data, while instantaneous distributions refer to 1 min time intervals. Thus during $\mathrm{BB}$ (NBB) intervals the presence of 1 min time intervals for which precipitation is almost exclusively of orographic (stratiform) origin is possible. Even more complicated situations can arise where both the orographic and stratiform mechanisms of drop production can operate simultaneously with different relative strengths. Here, we look for a metric which is capable of identifying $1 \mathrm{~min}$ time intervals where precipitation occurred mainly through an orographic mechanism. One of the advantages of this eventual metric with respect to the radar echoes used by Martner et al. (2008) would be its $1 \mathrm{~min}$ time resolution versus the $30 \mathrm{~min}$ resolution of the radar technique. The results of Fig. 4 indicate that a sharp difference exists between NBB and BB data sets at Bodega Bay and Cazadero for the right and left gradients. Therefore we use this variable to build a "steepness-orographic filter". One could envision a different metric, however the proposed metric has, in our opinion, some remarkable properties.

Given a renormalization time interval $I$ (of length $1 \mathrm{~min}$ in our case) of a dataset belonging to either the $\mathrm{s} 0, \mathrm{~s}+1$, or $\mathrm{s}+2$ skewness classes, the steepness-orographic filter operates as follows

$$
\left\{\begin{array}{l}
I \text { is steep if } \nabla_{L, I} \leq-9.2 \quad \text { OR } \nabla_{R, I} \leq-4 \\
I \text { is not steep if } \nabla_{L, I}>-9.2 \text { AND } \nabla_{R, I}>-4
\end{array} .\right.
$$

The values -9.2 and -4 are the $5 \%$ quantile of the left and right gradients for the DRW stratiform dataset. Thus this filter labels as "of strong orographic nature" the precipitation inside all renormalization time intervals whose left and right gradients qualify as statistically "uncommon" (5\% quantile) at Darwin (which is taken as reference data set for absence of orographic effect). When applied to the s012-BBY-NBB data set, 1428/2325 ( $661.41 \%)$ renormalization time intervals are classified as steep, while for the CZD-NBB data set the ratio is $2381 / 3213(\simeq 74.10 \%)$. For the data sets with bright band signature we find a ratio of $251 / 3004(\simeq 8.35 \%)$ for BBY-BB, and of 1149/3492 ( $\simeq 32.90 \%)$ for CZD-BB.

We now consider where the steep and not-steep portions of a dataset reside in the $\log _{10}\left(N_{I}\right) \mu_{I} \sigma_{I}$-plane. The average $\mu_{I}$ and the standard deviation $\sigma_{I}$ of the drop diameters are the only two parameters involved in the renormalization procedure, Eq. (1). The logarithm of the drop count $N_{I}$, instead of the drop count itself, is chosen for a better visualization. These three parameters are the "main" variables adopted in our statistical description of the rainfall phenomenon: e.g. we have shown in Ignaccolo and De Michele (2010) that rainfall rate classes (Tokay and
Short, 1996) occupy almost non overlapping volumes in the $\log _{10}\left(N_{I}\right) \mu_{I} \sigma_{I}$-plane. One remarkable property of the steepness filter is that the steep and not-steep portions of a data base (s012-BBY-NBB, s012-BBY-BB, s012-CZD-NBB, and s012-CZD-BB in our case) occupy two non overlapping volumes in the $\log _{10}\left(N_{I}\right) \mu_{I} \sigma_{I}$-plane. The $\mu_{I} \log _{10}\left(N_{I}\right)$-plane projections, left column of Fig. 5, indicate that none of the not-steep (blue crosses) renormalization time intervals have an average diameter $\mu_{I} \lesssim 0.5 \mathrm{~mm}$, while steep renormalization time intervals (red squares) are mostly concentrated in the region $\mu_{I} \lesssim 0.5 \mathrm{~mm}$ although some have a larger value of the average diameter. This overlap between steep and notsteep intervals is not present in the $\mu_{I} \sigma_{I}$-plane projections, right column of Fig. 5, where red squares and blue crosses represent two matching puzzle pieces: steep renormalization time intervals with an average diameter $\mu_{I} \gtrsim 0.5 \mathrm{~mm}$ have a standard deviation $\sigma_{I}$ which is either larger or smaller than that of not-steep time intervals indicating a "strong correlation" between $\mu_{I}, \sigma_{I}$ and the "steepness" of the instantaneous probability density $p_{G, I}(D)$.

Another remarkable property of the steepness-orographic filter is that it eliminates the right fatter tail discrepancies for the probability density $p_{G}\left(D_{R}\right)$ of the renormalized drop diameter $D_{R}$, Eq. (2), observed in Fig. 2 in the case of the $\mathrm{s} 0, \mathrm{~s}+1$, and $\mathrm{s}+2$ skewness class subsets of NBB rain periods. Figure 6 shows, left columns, probability densities $p_{G}\left(D_{R}\right)$ for the skewness class $\mathrm{s} 0, \mathrm{~s}+1$, and $\mathrm{s}+2$ sub sets of the not-steep portions of the s012-BBY-NBB and s012-CZDNBB data sets, together with the corresponding densities for BBY-BB and CZD-BB. There are no fatter tails and the densities are remarkably similar. Although s012-BBY-BB and and s012-CZD-BB have steep renormalization time intervals their contribution to the tails is not relevant and their inclusion or not does not alter the densities. If we compare the densities BBY-BB and CZD-BB with the steep portions of the s012-BBY-NBB and s012-CZD-NBB data sets, right column of Fig. 6, we see again the right fatter tail discrepancies.

It may seem counterintuitive that steeper distributions result in a fatter tail. However it is not so. The left and right gradient $\nabla_{L, I}$ and $\nabla_{R, I}$ are defined for the instantaneous probability density $p_{G, I}(D)$ while the fatter right tail occurs for $p_{G}\left(D_{R}\right)$ which is an average, Eq. (2), of single instantaneous renormalized densities $p_{G, I}\left(D_{R}\right)$. The left and right gradients are not preserved by the renormalization procedure. Using Eq. (1) together with Eq. (3) we see that the left and right gradients for the densities $p_{G, I}\left(D_{R}\right)$ differ from those relative to $p_{G, I}(D)$ by a multiplicative factor $\left(\sigma_{I}\right)^{2}$. Steep renormalization time intervals have a less than unity standard deviation, and have a smaller standard deviation than not-steep renormalization time intervals, with the exception of a fraction of the cases for which $\mu_{I} \gtrsim 0.5 \mathrm{~mm}$ (Fig. 5). Therefore the steepness of $p_{G, I}\left(D_{R}\right)$ is greatly reduced for steep, according to $p_{G, I}(D)$, renormalization time intervals while, in comparison, not-steep, according to $p_{G, I}(D)$, renormalization time intervals are subject to a 

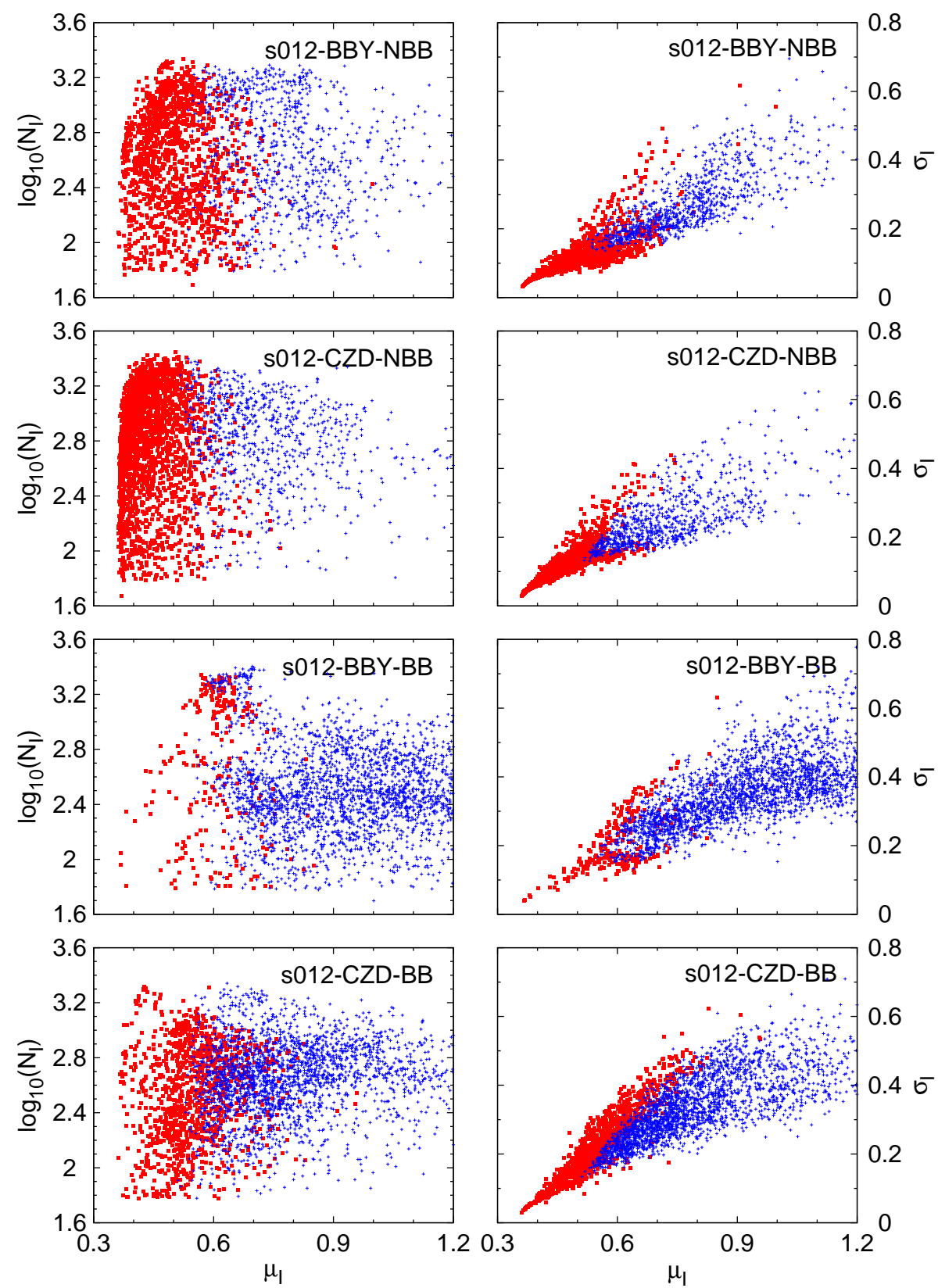

Fig. 5. Steep (red squares) vs. not-steep (blue crosses) portions, of BBY-NBB and CZD-NBB (the first four upper panels) and BBY-BB, CZD-BB (the four lower panels), both in the $\mu_{I} \log _{10}\left(N_{I}\right)$ plane (left panels), and in the $\mu_{I} \sigma_{I}$ plane (right panels).

much smaller reduction. A similar effect would be expected for the left tail, at least in the case of the BBY-NBB subsets as suggested by the cumulative density function $\mathcal{F}\left(\nabla_{L, I}\right)$, bottom panel of Fig. 4. However fatter left tails are not observed for the density of the BBY-NBB, or any other subsets. The rationale is that (1) overall the value of the left gradients are larger than those of the right gradients. (2) Very small values of the left gradients occur for $1 \mathrm{~min}$ time intervals for which the maximum of the density $p_{G, I}(D)$ and the mean values $\mu_{I}$ (see Figs. 4 and 5) are located predominantly in the first four diameter classes. As a consequence extremely negative values of the renormalized drop diameter, see Eq. (1), do not occur.

Finally, we see how the division in steep and not-steep subsets affects the cumulative density functions of the disdrometer diameter class of maximum density $j_{m, I}$, the class span $S_{I}$, the percentage occupancy $\% O_{I}$, Fig. 7 reports in the left column the results for the s012-BBY-NBB and s012-CZDNBB data sets, and in the right column the results for the s012-BBY-NBB and s012-CZD-NBB data sets. We see how 

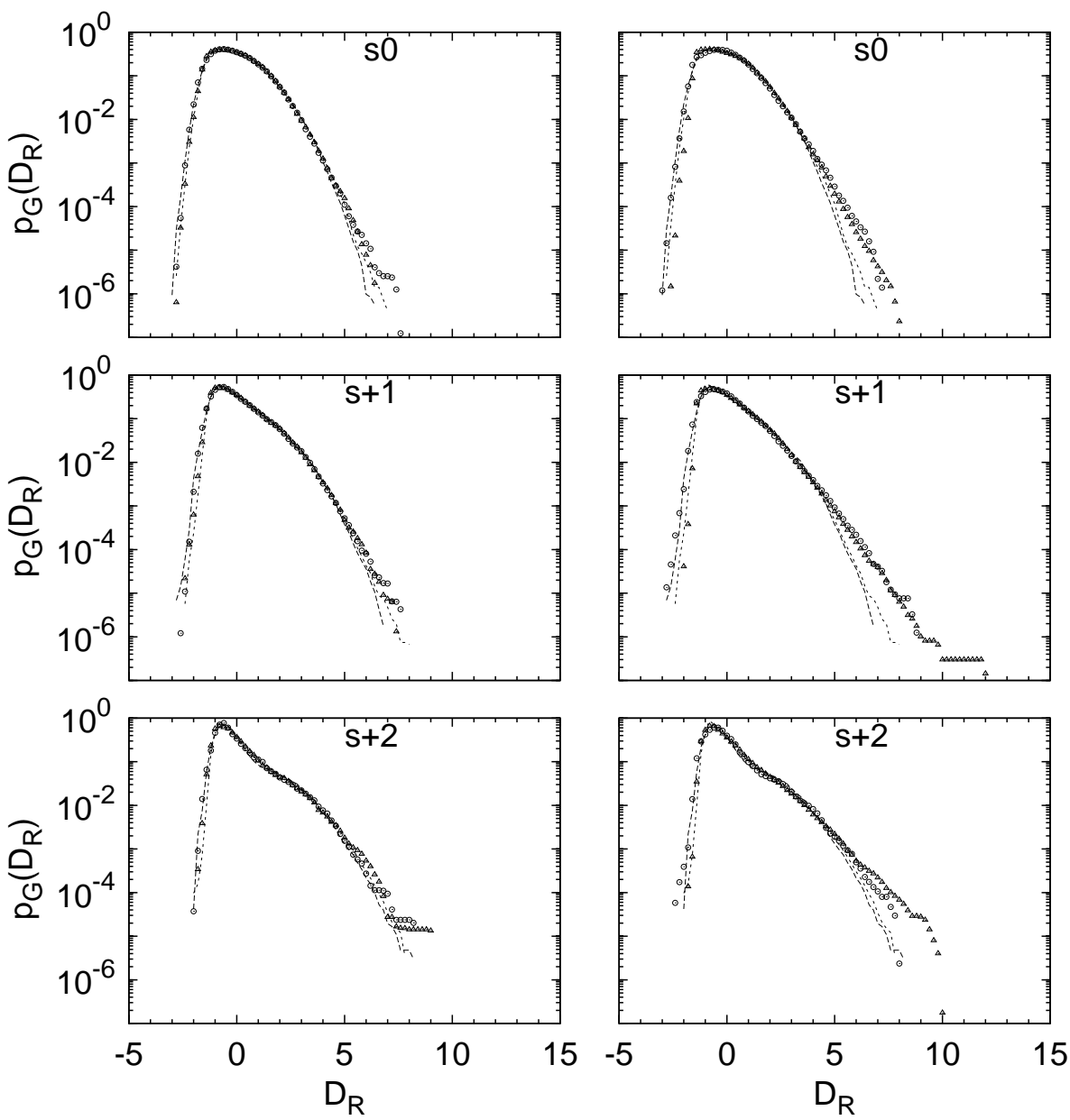

Fig. 6. $p_{G}\left(D_{R}\right)$ for the skewness classes $\mathrm{s} 0, \mathrm{~s}+1$, and $\mathrm{s}+2$ of not-steep (left panels) and steep (right panels) portions of the s012-BBY-NBB (circles) and s012-CZD-NBB (triangles) data sets, together with the corresponding densities for BBY-BB (long dashed line), CZD-BB (short dashed line).

in all cases the steep subsets (red and blue solid lines with not filled points) have instantaneous spectra which are more concentrated in the first four diameter classes, more narrow, and more apt to have a maximum in the first four diameter classes than the entire data sets (red and blue solid lines): in this sense the figure validates the use of the word "orographic" in "steepness-orographic filter". Not-steep subsets (red and blue solid lines with filled points) have instantaneous spectra whose properties are closer to those of the DRW stratiform database (solid black line).

\section{Conclusions}

The investigations presented in this work have led to several results.

Both in Bodega Bay and in Cazadero the distribution $\operatorname{Pr}\left(\gamma_{I}\right)$ of the skewness value of instantaneous raindrop spectra is strongly peaked (Fig. 1). Thus there is a most common distribution as in the case of Darwin explored in detail in the companion paper (Ignaccolo and De Michele, 2012). As for Darwin there are no dramatic differences in $\operatorname{Pr}\left(\gamma_{I}\right)$ between data sets with or without a bright band signature, even if the absence of a bright band echo in Darwin is associated with convective precipitation, while in Bodega Bay and in Cazadero to the occurrence of orographic precipitation.

Differences are present for the renormalized spectra $p_{G}\left(D_{R}\right)$ relative to different skewness class subsets of bright band and non bright band rain periods (Fig. 2). In the latter case the subsets relative to the skewness classes $\mathrm{s} 0, \mathrm{~s}+1$, and $\mathrm{s}+2$ have a fatter right tail. These differences are not present in Darwin when one compares stratiform (bright band) to convective (no bright band) precipitation. In Darwin stratiform or convective instantaneous renormalized spectra with the "same" (in the sense of skewness class) value of skewness have a common shape. This is not true in Bodega Bay and in Cazadero as NBB spectra differ from BB spectra in spite of having the same skewness. 

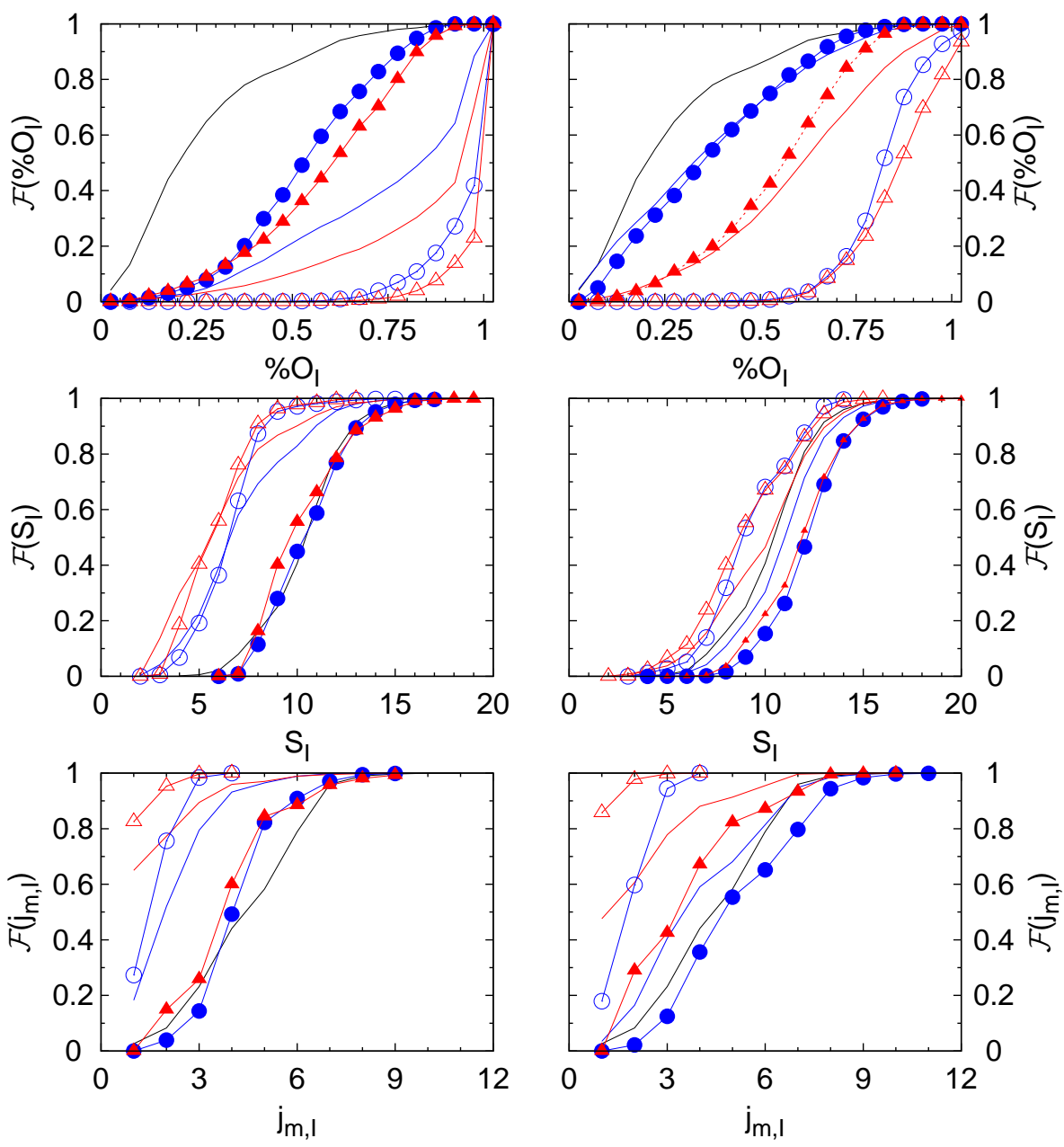

Fig. 7. Cumulative density functions of the following variables: the percentage occupancy $\% O_{I}$ (top panels), the class span $S_{I}$ (middle panels), and the disdrometer diameter class where the maximum density $j_{m, I}$ (bottom panels) occurs. The right (left) column refers to NBB (BB) sets: s012-BBY-NBB (s012-BBY-BB) solid blue line, s012-CZD-NBB (s012-CZD-BB) solid red line, steep subset of s012-BBY-NBB (s012-BBY-BB) solid blue line and blue filled circles, steep subset of s012-CZD-NBB (s012-CZD-BB) solid red line and blue filled circles, not-steep subset of s012-BBY-NBB (s012-BBY-BB) solid blue line and blue not filled circles, and not-steep subset of s012-CZD-NBB (s012-CZD-BB) solid red line and blue not filled circles.

NBB spectra are (Fig. 4) with respect to BB spectra more concentrated in the first four diameter classes $D \leq 0.715 \mathrm{~mm}$, narrower, more apt to have the maxima located in the first four diameter classes, and are steeper. These features agree with what are the known characteristic of orographic precipitation (Blanchard, 1953; Fujiwara, 1967) and the identification of NBB as rain periods during which condensation and precipitation are mostly orographic in nature (White at al., 2003; Martner et al., 2008).

We used the steepness parameters $\nabla_{L, I}$ and $\nabla_{R, I}$ to define a steepness-orographic filter which divides a data set in steep and not-steep subsets. A remarkable property of this filter is that steep and not-steep renormalization time intervals occupy two separate volumes in the $\log _{10}\left(N_{I}\right) \mu_{I} \sigma_{I}$-plane (Fig. 5). This means that one can redefine the steepnessorographic filter in terms of the parameters $N_{I}, \mu_{I}$, and $\sigma_{I}$ (since no appreciable overlap is observed between the steep and not-steep volume projections on the $\mu_{I} \sigma_{I}$-plane, one could redefine the steepness-orographic filter in terms of $\mu_{I}$ and $\sigma_{I}$ only). Another remarkable feature is that the filtering procedure eliminates the discrepancies (see Fig. 6) observed for renormalized spectra $p_{G}\left(D_{R}\right)$ relative to different skewness classes (s0, s +1 , and $\mathrm{s}+2)$ subsets of bright band and non bright band rain periods.

It is worth clarifying in which sense the filter defined in Eq. (4) is an "orographic" filter. Renormalization time intervals denoted as steep are time intervals during which precipitation is almost exclusively of orographic origin (Figs. 4, 5, and 7). This does not mean that during not-steep time intervals orographic precipitation does not occur but just that it is not predominant. 

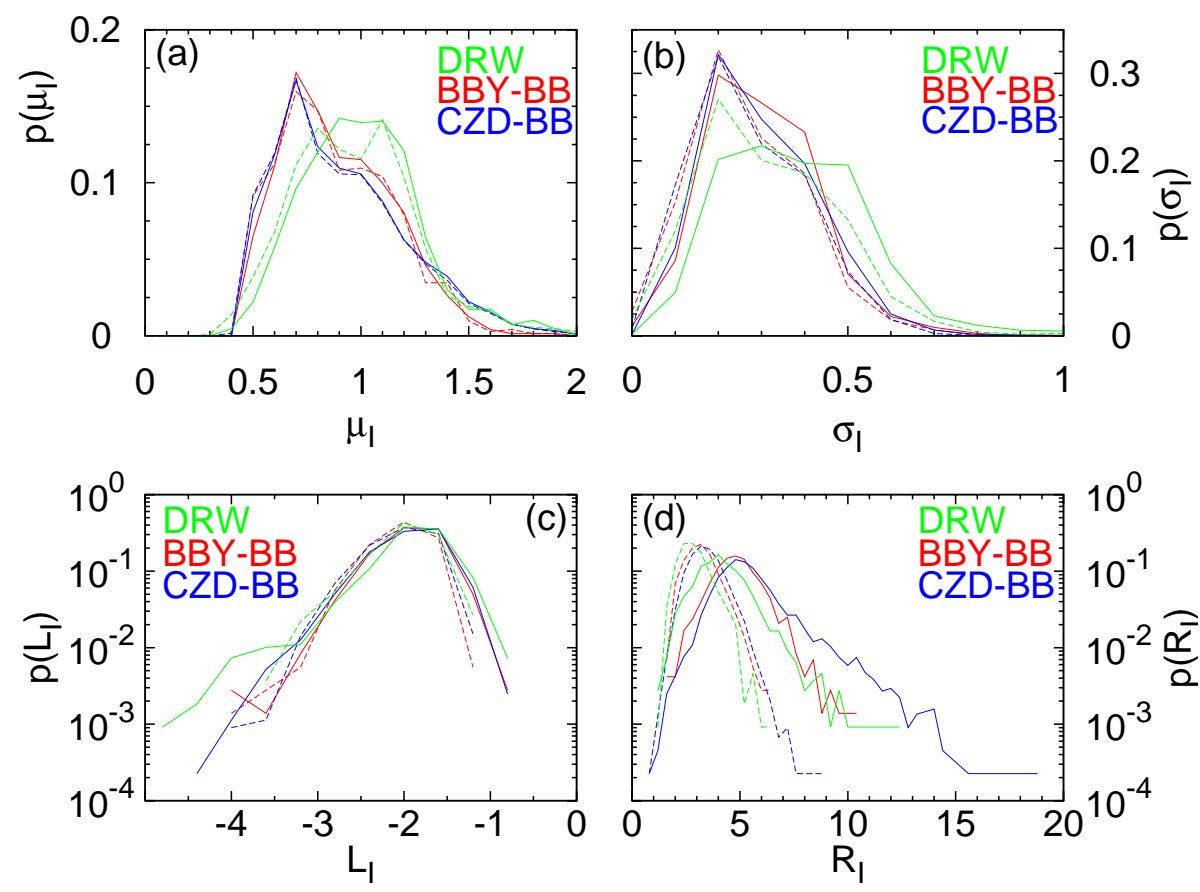

Fig. A1. The probabilities $p\left(\mu_{I}\right)$, panel (a), $p\left(\sigma_{I}\right)$, panel (b), $p\left(L_{I}\right)$, panel (c), and $p\left(R_{I}\right)$, panel (d), for DRW database and the BBY-BB and CZD-BB databases. Solid lines with outliers drop counts, dashed lines without.

Finally we want to comment on what may cause the shift in the location of the maxima of $\operatorname{Pr}\left(\gamma_{I}\right)$ observed in Fig. 1. After all, bright band (stratiform) precipitation at Bodega Bay and Cazadero should have the same characteristic as that at Darwin. A possibility is that the difference in peak location is due to the fact that at Darwin the precipitation is of monsoon origin while the data bases considered at Bodega Bay and Cazadero refer to winter storms arriving from the Pacific Ocean: synoptic hypothesis. However, we think that the evidence presented here suggests another hypothesis: the orographic hypothesis. The shift may be due to the presence of orographic precipitation. In fact even in the presence of the bright band signature (the presence of a bright band echo is inferred using $30 \mathrm{~min}$ averaged radar data) orographic precipitation may occur and be either predominant leading to "steep" time intervals $(\simeq 8.35 \%$ of BBY-BB and $\simeq 32.90 \%$ of CZD-BB data sets are steep intervals) or simply occur in superposition, without being predominant, to the rain of stratiform origin (White at al., 2003; Martner et al., 2008). In the latter case we expect an enhancement of the drop population in the first four classes $\left(\% O_{I}\right)$ and larger possibility that the maximum of instantaneous spectra is located in the first four classes $\left(j_{m, I}\right)$, and the span $\left(S_{I}\right)$ of the spectra to remain essentially unaltered. This is precisely what we observe when we compare (Figs. 4 and 7) the cumulative density functions for the variables $\% O_{I}, j_{m, I}$, and $S_{I}$ of BB data set or not-steep subsets of any set with the respective cumulative density functions for stratiform precipitation at Darwin. Moreover the fact the Cazadero is in the Coastal Range Mountain, while Bodega Bay is on the coast would suggest a stronger orographic effect in Cazadero than in Bodega Bay explaining why the shift in skewness values is larger at Cazadero.

\section{Appendix A}

\section{The effect of outliers drop counts}

Here we discuss the effect of outliers drop counts on: (1) the renormalization parameters, $\mu_{I}$, the mean drop diameter, and $\sigma_{I}$, the standard deviation of the drop diameter; (2) the left and right tail of the probability density function at ground $p_{G}\left(D_{R}\right)$ for the renormalized drop diameter $D_{R}$ (Eq. 2). In this latter case we will introduce two extra parameters: the minimum $L_{I}$ and maximum $R_{I}$ value of the renormalized drop diameter $D_{R}$ inside the renormalization time interval $I$. These parameters are calculated applying the first relation of Eq. (1) to the left (right) border of the smallest (largest) diameter class with a non zero count.

To quantify the effect of the outliers drop counts we compare the values of the parameters $\mu_{I}, \sigma_{I}, L_{I}, R_{I}$, and the probability densities $p_{G}\left(D_{R}\right)$ with and without outliers. Figures A1-A3 show the results of these analyses. In Fig. A1 we consider the entire DRW database and the subsets of BBY and CZD databases with negligible or small orographic perturbation (CZD-BB and BBY-BB), and plot the probabilities 

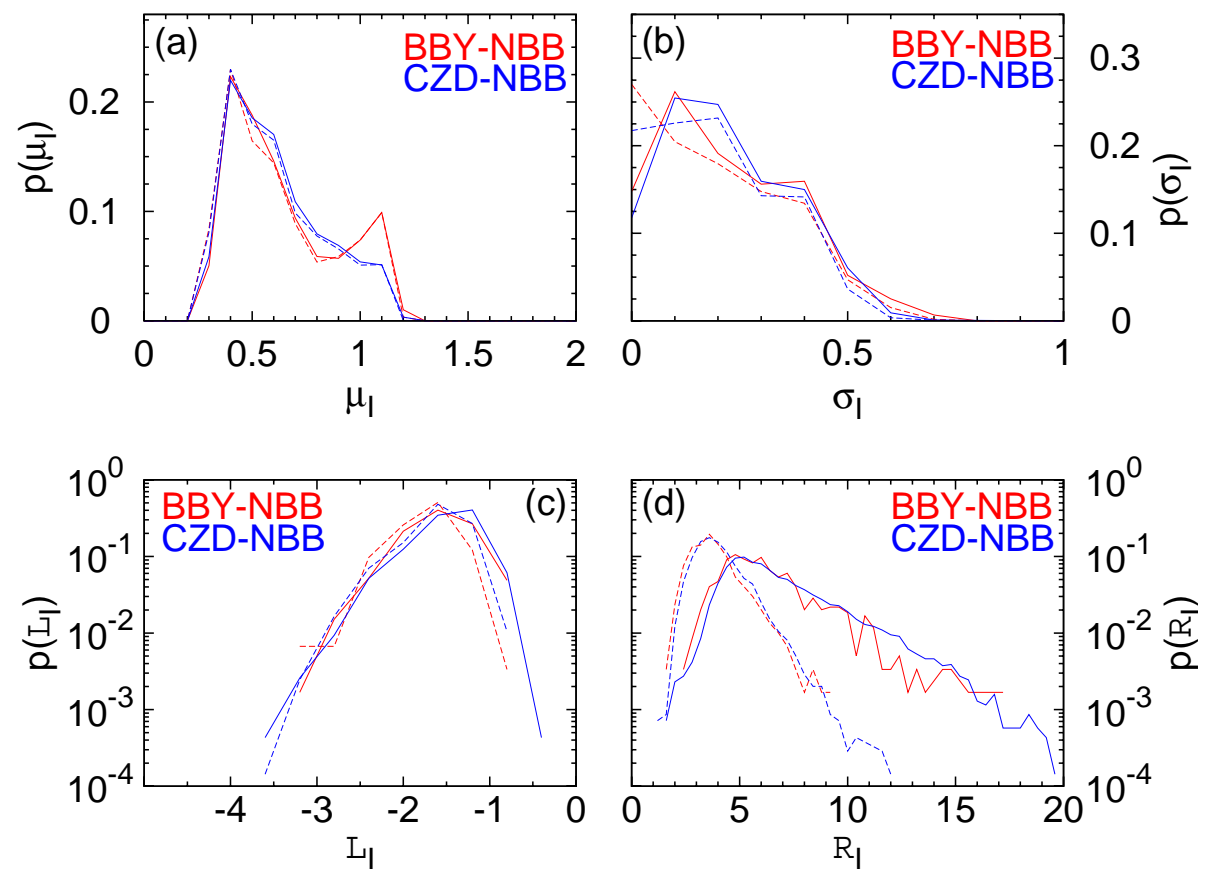

Fig. A2. The probabilities $p\left(\mu_{I}\right)$, panel (a), $p\left(\sigma_{I}\right)$, panel (b), $p\left(L_{I}\right)$, panel (c), and $p\left(R_{I}\right)$, panel (d), for the BBY-NBB and CZD-NBB databases. Solid lines with outliers drop counts, dashed lines without.
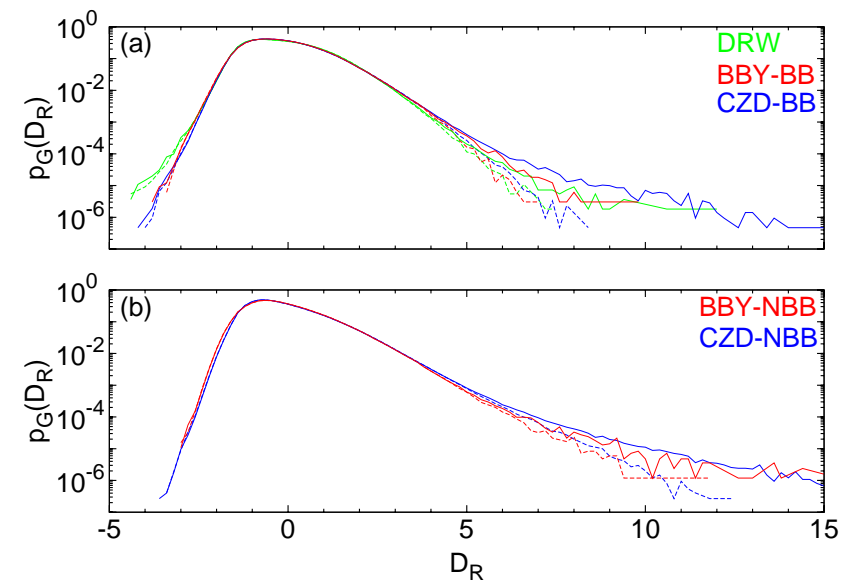

Fig. A3. The probability density $p_{G}\left(D_{R}\right)$ for the entire DRW database and the BBY-BB and CZD-BB databases - panel (a), and for the BBY-NBB and CZD-NBB databases - panel (b). Solid lines with outliers drop counts, dashed lines without.

of observing a particular value of the parameters $\mu_{I}, \sigma_{I}, L_{I}$, and $R_{I}$ prior (solid lines) and after (dashed lines) the removal of outliers drop counts. We see how the presence of outliers drop counts perturbs only lightly the distributions of the average drop diameter $\mu_{I}$, the standard deviation of drop diameters $\sigma_{I}$ the minimum renormalized drop diameter $L_{I}$, while it strongly affects the distribution of maximum renormalized drop diameter $R_{I}$ (CZD-BB). Similar results are obtained for the subsets of BBY and CZD databases with strong orographic perturbation (BBY-NBB and CZD-NBB) as depicted in Fig. A2. The "strong" effect on the maximum renormalized drop diameter $R_{I}$ is due to some instances where the largest diameter class with a non zero count is separated from the non gap region by more than 1 diameter class, i.e. the following drops count: 111710161221291686197500 01000 .

These results suggest that the right tail of the probability density $p_{G}\left(D_{R}\right)$ is heavily affected by outliers. This hypothesis is confirmed by the plots reported in Fig. A3. We see how the exceedingly slow decay (when compared to the DRW and the BBY-BB databases) of the right tail the probability $p_{G}\left(D_{R}\right)$ relative to the CZD-BB database (solid blue line of Fig. A3a), is due to the renormalization time intervals with outliers drop counts (in agreement of Fig. A1d). Once the outliers are removed from all the three data sets the probabilities $p_{G}\left(D_{R}\right)$ show the "same" (differences due to just sample fluctuations) decay. Figure A3b shows that the right tail of the probability $p_{G}\left(D_{R}\right)$ of CZD-NBB database has a slower decay than the one of BBY-NBB database when the outliers drop counts are included (solid line) and a better agreement when the outliers are excluded (dashed line), as expected from the results depicted in Fig. A2d.

Acknowledgements. We are thankful to C. R. Williams and the National Oceanic and Atmospheric Administration for the public availability of the data set. M. I. thankfully acknowledges the support of Accademia Nazionale dei Lincei (Italy) through "B. Segre" scholarship. C. D. M. thankfully acknowledges Comune di Milano (Italy) through BIODESCESA project.

Edited by: R. Uijlenhoet 


\section{References}

Blanchard, D. C.: Raindrop size-distribution in Hawaiian rains, J. Meteorol., 10, 457-473, 1953.

Campos, E. F.: On measurements of drop size distribution, Top. Meteor. Oceanog., 6, 24-30, 1999.

Campos, E. F., Zawadzki, I., Petitdidier, M., and Fernandez, W.: Measurement of raindrop size distributions in tropical rain at Costa Rica, J. Hydrol., 328, 98-109, 2006.

Fujiwara, M.: Raindrop size distribution in warm rain as measured in Hawaii, Tellus XIX, 3, 392-402, 1967.

Hazenberg, P., Nan, Y., Boudevillain, B., Delrieu, G., and Uijlenhoet, R.: Scaling of raindrop size distributions and classification of radar reflectivity rain rate relations in intense Mediterranean precipitation, J. Hydrol., 402, 179-192, 2011.

Houze, R. A.: Stratiform precipitation in regions of convection: a meteorological paradox?, B. Am. Meteorol. Soc., 78, 21792196, 1997.

Ignaccolo, M. and De Michele, C.: Statistical collapse of stratiform and convective drop diameter distributions at the ground, Geophys. Res. Lett., 37, L24402, doi:10.1029/2010GL045454, 2010.

Ignaccolo, M. and De Michele, C.: Skewness as measure of the invariance of instantaneous renormalized drop diameter distributions - Part 1: Convective vs. stratiform precipitation, Hydrol. Earth Syst. Sci., 16, 319-327, doi:10.5194/hess-16-319-2012, 2012.

Ignaccolo, M., De Michele, C., and Bianco, S.: The drop-like nature of rain and its invariant statistical properties, J. Hydrometeorol., 10, 79-95, 2009.

Joss, J. and Gori, E. G.: Shapes of raindrop size distributions, J. Appl. Meteorol., 17, 1054-1061, 1978.

Kingsmill, D. E., Neiman, P. J., Ralph, F. M., and White, A. B.: Synoptic and topographic variability of northern California precipitation characteristics in landfalling winter storms observed during CALJET, Mon. Weather Rev., 134, 2072-2094, 2006.

Konstinski, A. B. and Jameson, A. R.: Fluctuation properties of precipitation, Part I: on deviations of single-size drop counts from the Poisson distribution, J. Atmos. Sci., 54, 2174-2186, 1997.

Maki, M., Keenan, T. D., Sasaki, Y., and Nakamura, K.: Characteristics of the raindrop size distribution in tropical continental squall lines observed in Darwin, Australia, J. Appl. Meteorol., 40, 1393-1412, 2001.
Martner, B. E., Yuter, S. E., Allen, B. W., Matrosov, S. Y., Kingsmill, D. E., and Ralph, F. M.: Raindrop size distributions and rain characteristics in California coastal rainfall for periods with and without a radar bright band, J. Hydrometeorol., 9, 408425, 2008.

Neiman, P. J., Wick, G. A., Ralph, F. M., Martner, B. E., White, A. B., and Kingsmill, D. E.: Wintertime nonbrightband rain in California and Oregon during CALJET and PACJET: geographic, interannual and synoptic variability, Mon. Weather Rev., 133, 1199-1223, 2005.

Roe, G. H.: Orographic precipitation, Ann. Rev. Earth Pl. Sci., 33, 645-671, 2005.

Sauvageot, H. and Lacaux, J. P.: The shape of averaged drop size distributions, J. Atmos. Sci., 52, 1070-1083, 1995.

Sekhon, R. S. and Srivastava, R. C.: Doppler radar observations of drop-size distributions in a thunderstorm, J. Atmos. Sci., 28, 983-994, 1971.

Sempere Torres, D., Porrà, J. M., and Creutin, J. D.: A general formulation for raindrop size distribution, J. Appl. Meteorol., 33, 1494-1502, 1994.

Smith, J. A.: Marked point process models of raindrop-size distributions, J. Appl. Meteorol., 32, 284-296, 1993.

Testud, J., Oury, S., Black, R. A., Amayenc, P., and Dou, X.: The concept of normalized distribution to describe raindrop spectra: a tool for cloud physics and cloud remote sensing, J. Appl. Meteorol., 40, 1118-1140, 2011.

Tokay, A. and Short, D. A.: Evidence from tropical raindrop spectra of the origin of rain from stratiform versus convective clouds, J. Appl. Meteorol., 35, 355-371, 1996.

Uijlenhoet, R., Smith, J. A., and Steiner, M.: The microphysical structure of extreme precipitation as inferred from ground-based raindrop spectra, J. Atmos. Sci., 60, 1220-1238, 2003.

White, A. B., Neiman, P. J., Ralph, F. M., Kingsmill, D. E., and Ola, P., and Persson, G.: Coastal Orographic Rainfall Processes Observed by Radar during the California Land-Falling Jets Experiment, J. App. Meteorol., 4, 264-282, 2003.

Willis, P. T.: Functional fits to some observed dropsize distributions and parameterization of rain, J. Atmos. Sci., 41, 1648-1661, 1984. 